Bundesgesundheitsbl 2017 · 60:1298-1304 https://doi.org/10.1007/s00103-017-2631-9 Online publiziert: 11. September 2017

๑) Springer-Verlag GmbH Deutschland 2017
Bekanntmachung des Umweltbundesamtes

\section{Richtwert für Propan-1,2-diol (Propylenglykol) in der Innenraumluft}

\author{
Mitteilung des Ausschusses für \\ Innenraumrichtwerte
}

\section{Stoffidentifikation [6]}

Systematischer Name: Propan-1,2-diol Synonyme: 1,2-Propandiol Propylenglykol 1,2-Dihydroxypropan

EC-Nr.: 200-338-0

CAS-Nr: 57-55-6 Summenformel: $\mathrm{C}_{3} \mathrm{H}_{8} \mathrm{O}_{2}$ Strukturformel:<smiles>CC(O)CO</smiles>

\subsection{Physikalische und chemische Eigenschaften}

Propan-1,2-diol (ältere Bezeichnung Propylenglykol (PG)) ist eine hygroskopische, visköse, geruch- und farblose Flüssigkeit mit einem niedrigen Dampfdruck $[6,41]$.

$\begin{array}{ll}\text { Molekulargewicht: } & 76,094 \mathrm{~g} / \mathrm{mol} \\ \text { Schmelzpunkt: } & -60{ }^{\circ} \mathrm{C} \\ \text { Siedepunkt: } & 188,2{ }^{\circ} \mathrm{C} \\ \text { Dichte: } & 1,0361 \mathrm{~g} / \mathrm{cm}^{3} \\ & \text { bei } 20^{\circ} \mathrm{C} \\ \text { Dampfdruck: } & 20 \mathrm{~Pa} \text { bei } 25^{\circ} \mathrm{C} \\ \text { Wasserlöslichkeit: } & \text { vollständig } \\ & \text { mischbar } \\ \text { log } \mathrm{P}_{\text {Octaol/Wasser }}: & -0,912\end{array}$

Umrechnung (bei $20{ }^{\circ} \mathrm{C}$ ): $1 \mathrm{ml} / \mathrm{m}^{3}$ $=3,16 \mathrm{mg} / \mathrm{m}^{3}, 1 \mathrm{mg} / \mathrm{m}^{3}=0,32 \mathrm{ml} / \mathrm{m}^{3}$

\subsection{Anwendung}

Beim PG handelt es sich mit einem jährlichen Produktionsvolumen von ca. 3 Millionen Tonnen um eine in hohen Quantitäten produzierte organische Che- mikalie [45]. Aufgrund seiner physikalisch-chemischen Charakteristika wird PG insbesondere in der Herstellung von Polyesterharzen, Enteisungs- und Wärmetransportmitteln, Kosmetika (z. B. in Feuchtigkeitscremes, Lösemitteln oder Duftstoffen), sogenannten Mitteln zur Verbesserung der Raumluft, Pharmazeutika, Pestiziden, Farben, Beschichtungssystemen, Tierfutter und in Nahrungsmitteln eingesetzt [12, 45]. Darüber hinaus wird es als Befeuchtungsmittel von Tabakprodukten, in sogenannten Liquids von E-Zigaretten, als Komponente in hydraulischen Bremsflüssigkeiten und in Weichmachern benutzt. Eine Zusammenstellung der ungefähren Verwendung gibt die • Tab. 1.

\section{Exposition}

Aufgrund der vielfältigen Anwendungen von PG ist eine orale und dermale Exposition über Nahrungsmittel bzw. körper- nahe Verbraucherprodukte und eine inhalative Aufnahme, insbesondere aus der Innenraumluft, zu erwarten.

\subsection{Innenraumluft}

In der $\bullet$ Tab. 2 sind Ergebnisse zu PGMessungen in der Luft ausgewählter Innenräume (Wohnungen, Klassenräume, Büros) zusammengestellt. In einer Untersuchung von 3562 Aufenthaltsräumen in Deutschland (u.a. Wohnungen, Kindertagestätten, Schulen, Büroräumen) zwischen 2006 und 2012 ließ sich PG bei einer Bestimmungsgrenze von $1 \mu \mathrm{g} / \mathrm{m}^{3}$ in $47 \%$ der Innenräume nachweisen, der Median betrug $2 \mu \mathrm{g} / \mathrm{m}^{3}$, das 95 . Perzentil $27 \mu \mathrm{g} / \mathrm{m}^{3}$ und der Maximalwert $870 \mu \mathrm{g} / \mathrm{m}^{3}$ [19].

Kurzzeitig hohe PG-Gehalte in der Innenraumluft wurden in früheren Untersuchungen bei der Anwendung sogenannter Nebelfluide im Rahmen von z. B. Theateraufführungen berichtet. In den Jahren
Tab. 1 Verwendungsbereiche von Propylenglykol (nach [DFG 2007])

\begin{tabular}{ll} 
Verwendung & $\begin{array}{l}\text { \% des Jahres- } \\
\text { Produktionsvolumens (ca.) }\end{array}$ \\
\hline $\begin{array}{l}\text { Ungesättigte Polyesterharze (Harz, Monomer) } \\
\begin{array}{l}\text { Nahrungsmittel, Pharmazeutika, Kosmetik und Pflegeartikel } \\
\text { (Befeuchtungsmittel, Weichmacher) }\end{array}\end{array}$ & $38-40$ \\
$\begin{array}{l}\text { Gefrierschutz, Flugzeugenteisungsmittel, } \\
\text { industrielle Schmiermittel (Schmiermittel, Kühlschmierstoff) }\end{array}$ & $10-13$ \\
$\begin{array}{l}\text { Flüssigwaschmittel (Dispergiermittel) } \\
\text { Tiernahrung (Befeuchtungsmittel) }\end{array}$ & $9-15$ \\
$\begin{array}{l}\text { Tabakzusatzstoff (Befeuchtungsmittel) } \\
\text { Farben und Beschichtungen (Lösungsmittel) }\end{array}$ & $5-6$ \\
Sonstiges (diverse Weichmacher) & $3-4$ \\
& 4 \\
\hline
\end{tabular}


Tab. 2 Vorkommen von Propylenglykol in der Luft von Wohnräumen, Klassenräumen und Büroräumen in $\mu \mathrm{g} / \mathrm{m}^{3}$

\begin{tabular}{|c|c|c|c|c|c|}
\hline Referenz & Anzahl & Median & 95. Perzentil & Maximum & Beschreibung \\
\hline \multicolumn{6}{|l|}{ Wohninnenräume } \\
\hline $\begin{array}{l}\text { Schleibinger et al. } 2001 \\
\text { [35] }\end{array}$ & 90 & $<4$ & 36 & 165 & $\begin{array}{l}\text { Innenräume, } \\
\text { 1988-1999 }\end{array}$ \\
\hline $\begin{array}{l}\text { Hodgson et al. } 2000 \\
\text { [18] }\end{array}$ & 4 & $2,2^{2}$ & - & 12 & $\begin{array}{l}\text { Neue Häuser, } \\
\text { 1997/98 }\end{array}$ \\
\hline Zhu et al. 2004 [51] & 9 & $8,7^{1}$ & - & 25 & Häuser \\
\hline Choi et al. 2010 [8] & 390 & 5,5 & - & 49 & Kinderzimmer \\
\hline $\begin{array}{l}\text { Hofmann et al. } 2013 \\
\text { [19] }\end{array}$ & 673 & 2,5 & 25 & - & $\begin{array}{l}\text { Wohnräume, } \\
\text { 2006-2012 }\end{array}$ \\
\hline \multicolumn{6}{|l|}{ Schulen } \\
\hline $\begin{array}{l}\text { Fromme et al. } 2008 \\
\text { [14] }\end{array}$ & 165 & $<0,2$ & 12 & 32 & $\begin{array}{l}\text { Klassenräume, } \\
\text { 2004-2005 }\end{array}$ \\
\hline $\begin{array}{l}\text { Hofmann et al. } 2013 \\
\text { [19] }\end{array}$ & 493 & 0,5 & 23 & - & $\begin{array}{l}\text { Klassenräume, } \\
\text { 2006-2012 }\end{array}$ \\
\hline \multicolumn{6}{|l|}{ Bürogebäude } \\
\hline $\begin{array}{l}\text { Hofmann et al. } 2013 \\
\text { [19] }\end{array}$ & 1743 & 2,0 & 29 & - & $\begin{array}{l}\text { Büroräume, } \\
\text { 2006-2012 }\end{array}$ \\
\hline $\begin{array}{l}\text { Salonen et al. } 2009 \\
\text { [34] }\end{array}$ & 176 & $7,0^{1}$ & - & 280 & $\begin{array}{l}\text { Bürogebäude, } \\
\text { 2001-2003 }\end{array}$ \\
\hline
\end{tabular}

1991-1993 fand das US-amerikanische National Institute for Occupational Safety and Health bei drei Theateraufführungen PG-Gehalte zwischen $<0,01$ und $1,9 \mathrm{mg} /$ $\mathrm{m}^{3}$ [40]. Auch Neumann et al. (1996) [30] konnten nach einer Verneblung auf der Bühne eine Konzentration von $30 \mathrm{mg}$ $\mathrm{PG} / \mathrm{m}^{3}$ messen, wobei die Gehalte in der ersten Reihe im Zuschauerraum noch bei $8 \mathrm{mg} \mathrm{PG} / \mathrm{m}^{3}$ lagen. In einer weiteren USamerikanischen Studie wurden 1997-99 auf 16 Bühnen bei Nebelanwendungen Konzentration von $0,1-37 \mathrm{mg} / \mathrm{m}^{3}$ mit einer Spitzenkonzentration von $160 \mathrm{mg} / \mathrm{m}^{3}$ an Gesamtglykolen (Summe Propylenglykol, Butylenglykol, Di- und Triethylenglykol) beobachtet [27].

PG ist auch ein wesentlicher Bestandteil der sogenannten Liquids von E-Zigaretten und kann im exhalierten Aerosol beim Dampfen von E-Zigaretten gefunden werden $[37,26]$. In einer $30 \mathrm{~m}^{3}$ Testkammer traten je nach Typ der E-Zigarette Spitzenkonzentrationen von 1,4 oder $2,2 \mathrm{mg}$ $\mathrm{PG} / \mathrm{m}^{3}$ auf [15]. Schober et al. (2014) [36] fanden in einem Büroraum unter üblichen Lüftungsbedingungen während 6 jeweils zweistündiger Rauchsitzungen PG-Gehalte von 110-395 $\mu \mathrm{g} / \mathrm{m}^{3}$; ohne das Rauchen von E-Zigaretten lagen die PG-Ge- halte unter $0,04 \mu \mathrm{g} / \mathrm{m}^{3}$. In einem kleinen Besprechungsraum, in dem 3 Personen über 165 Minuten E-Zigaretten dampften, wurde in diesem Zeitraum ein Gehalt von $204 \mu \mathrm{g} P G / \mathrm{m}^{3}$ in der Innenraumluft gemessen, während vor der Rauchsitzung nur Konzentrationen von $<0,5 \mu \mathrm{g} / \mathrm{m}^{3}$ beobachtet wurden [31]. Beim Verdampfen von PG aus E-Zigaretten liegt PG als Aerosol in der Innenraumluft vor, wobei das Maximum von Partikeln Durchmesser zwischen 20-30 nm aufweist [36, 37].

\subsection{Nahrungsmittel}

In der EU sind für PG (E 1520) als maximale Obergrenze im Endnahrungsmittel $1000 \mathrm{mg} / \mathrm{kg}$, z. T. auch $3000 \mathrm{mg} / \mathrm{kg}$, zulässig [11].

Auf der Basis von Daten aus der Industrie schätzte das Joint FAO/WHO Expert Committe on Food Additives (JECFA) 1995 eine tägliche Zufuhr über Lebensmittel von $0,5 \mathrm{mg}$ PG/kg Körpergewicht (KG) ab [23]. Daneben sind nur zwei neuere Abschätzungen zur nahrungsbedingten Aufnahme aus Japan und Korea veröffentlicht worden. In Japan wird für Erwachsene und Kinder im Jahr 2000 eine tägliche Aufnahme von 0,18 bzw. 0,29 mg/ kg KG abgeschätzt [20]. In einer 2012-14 durchgeführten nationalen Verzehrsstudie in Korea lag die mittlere tägliche PGAufnahme bei $0,52 \mathrm{mg} / \mathrm{kg} \mathrm{KG}$ und das 95 . Perzentil bei 2,4 mg PG/kg KG [25]. Für Deutschland liegen keine Daten zur nahrungsbedingten Aufnahme von PG vor.

\section{Toxikokinetik}

Für den Menschen liegen keine geeigneten toxikokinetischen Daten nach inhalativer Aufnahme von PG vor. Einen gewissen Hinweis liefert eine ältere Untersuchung von 6 gesunden Probanden und 8 Patienten mit einer Lungenerkrankung, die über eine Stunde in einem Zelt einem technetiummarkierten Aerosol (medianer Durchmesser 4,8-5,3 $\mu \mathrm{m}$ ), das $10 \%$ PG enthielt, ausgesetzt waren [5]. Weniger als $5 \%$ des radioaktiven Technetiums wurden systemisch aufgenommen, während $90 \%$ sich zuerst im Nasenrachenraum absetzten und dann im Magen gefunden wurden. Die inhalative Aufnahme von PG konnte mit dieser Methodik nicht ermittelt werden, aber die Autoren gehen vor dem Hintergrund der Ergebnisse von einer geringen Aufnahme von PG-Aerosol über die Lunge aus.

In neueren Studien wurde die inhalative Aufnahme von PG mittels eines KapillarAerosolgenerators an Ratten und Hunden untersucht. Die Mediane der aerodynamischen Massendurchmesser (MMAD) der Aerosole lagen zwischen 0,9 und 2,3 $\mu \mathrm{m}$. In beiden Spezies erfolgte die Absorption des PG-Aerosols bei pulmonaler Exposition sehr schnell und das Konzentrationsgleichgewicht zwischen Lunge und Plasma stellte sich innerhalb von 50 Minuten für alle PG-Dosen ein. Die proportionalen Anstiege der Lungen- und Plasma-PGKonzentrationen mit den nominalen Dosen weisen darauf hin, dass die Absorption eine Reaktion erster Ordnung ist [44].

Im Rahmen von Arzneimitteluntersuchungen mit PG als Trägermaterial zeigte sich, dass oral verabreichtes PG schnell aus dem Magen-Darm-Trakt aufgenommen und bereits nach ca. 1 Stunde sein Plasmamaximum erreicht [49]. Tierexperimentelle Studien an Ratten und Kaninchen kommen zu vergleichbaren Ergebnissen $[28,29]$.

Propan-1,2-diol wird mehrstufig metabolisiert $[3,10]$, indem es zunächst durch 
die NAD-abhängige Alkoholdehydrogenase $(\mathrm{ADH}) \mathrm{zu}$ 2-Hydroxypropanal (DLLactaldehyd) oxidiert wird. Der zweite Schritt besteht in der Oxidation des 2-Hydroxypropanals zur 2-Hydroxypropansäure bzw. zum 2-Hydroxypropanat (Lactat) durch die Aldehyddehydrogenase (ALDH) oder in einem Nebenweg über 2-Oxopropanal (Methylglyoxal) durch die $\mathrm{ADH}$ und entsprechende Glyoxalase ebenfalls zur 2-Hydroxypropansäure bzw. zum D-Lactat.

Beim Menschen betrug die Eliminationshalbwertszeit nach ein- oder mehrmaliger oraler Gabe von 20,7 bzw. 41,4 g PG 3,8-4,1 Stunden [49]. Nach i.v.-Gabe an 6 Patienten wurde eine Halbwertszeit von 2,3 Stunden beobachtet [38]. Tierexperimentelle Untersuchungen an Ratten und Hunden weisen auf eine kapazitätsbegrenzte Elimination hin, da die interne Belastung mit steigender nominaler Dosis (Ratten: bis $0,4 \mathrm{~g} / \mathrm{kg}$ und Hunde: bis $0,3 \mathrm{~g} / \mathrm{kg}$ ) überproportional zunahm. Die terminalen Plasma- und Lungenkonzentrationszeitprofile lassen vermuten, dass die Elimination eine Reaktion nullter Ordnung ist [44]. Die Ausscheidung der 2-Hydroxypropansäure erfolgt beim Menschen zu ca. $45 \%$ in glucuronidierter Form über die Niere [2]. Tierexperimentelle Studien kommen zu vergleichbaren toxikokinetischen Daten [50].

\section{Gesundheitliche Wirkungen}

Die gesundheitliche Wirkung von PG nach oraler oder dermaler Applikation ist gut untersucht $[3,10]$. Die NOAELs in den oralen Studien liegen im Allgemeinen im g/kg KG-Bereich. Unter okklusiven Bedingungen zeigt PG dermal irritative Wirkungen. Eine hautsensibilisierende Wirkung von Propylenglykol konnte in systematischen Untersuchungen weder beim Menschen noch am Tier gezeigt werden. Die Ergebnisse deuten insgesamt auf ein geringes Sensibilisierungspotential von PG bei intakter Haut hin, auch wenn vereinzelt hautsensibilisierende Reaktionen beobachtet worden sind, insbesondere bei Dermatitis-Patienten.

Zur gesundheitlichen Wirkung eingeatmeten PGs liegen nur wenige Untersuchungen vor, die im Folgenden kurz beschrieben werden. Untersuchungen $\mathrm{zu}$ einer atemwegssensibilisierenden Wirkung von PG fehlen bislang [10].

Bundesgesundheitsbl 2017·60:1298-1304 DOI 10.1007/s00103-017-2631-9

○) Springer-Verlag GmbH Deutschland 2017

Bekanntmachung des Umweltbundesamtes

Richtwert für Propan-1,2-diol (Propylenglykol) in der Innenraumluft. Mitteilung des Ausschusses für Innenraumrichtwerte

\section{Zusammenfassung}

Zum Schutz der Gesundheit der Bevölkerung setzt der Ausschuss für Innenraumrichtwerte Richtwerte für die Innenraumluft fest. Für eine gesundheitliche Bewertung von Propan-1,2-diol in der Luft liegen bisher keine hinreichend aussagekräftigen Humanstudien vor. In einer gut dokumentierten und als zuverlässig eingestuften subchronischen Inhalationsstudie an Ratten wurden nasale Blutungen beobachtet. Aus dieser Studie ergibt sich eine LOAEC für kontinuierliche Exposition von $28,6 \mathrm{mg} / \mathrm{m}^{3}$ Propan-1,2-diol

\begin{abstract}
für den Endpunkt nasale Blutungen. Mit einem Faktor von 2 zur Extrapolation von subchronisch zu chronischer Exposition, von 2,5 für Interspeziesunterschiede und von 10 für interindividuelle Variabilität ergibt sich ein Richtwert II (Gefahrenwert) von 0,6 mg/ $\mathrm{m}^{3}$ Propan-1,2-diol und ein Richtwert I (Vorsorgewert) von $0,06 \mathrm{mg} / \mathrm{m}^{3}$ Raumluft.
\end{abstract}

Schlüsselwörter

Propan-1,2-diol • Propylenglykol • Reizwirkung - Innenraumluft • Richtwert

\section{Indoor air guide value for propan-1,2-diol (propylene glycol). Communication from the Committee on Indoor Guide Values}

\section{Abstract}

The Committee on Indoor Guide Values is issuing indoor air guide values to protect public health. At present, no suitable human studies are available for health evaluation of propan-1,2-diol in indoor air. In a well-documented subchronic inhalation animal study with rats, assessed as reliable, nasal hemorrhages were observed. This study leads to a LOAEC of $28.6 \mathrm{mg} / \mathrm{m}^{3}$ propan-1,2-diol for continuous exposure for the endpoint nasal hemorrhages. By applying a conversion factor of 2 from subchronic to chronic exposure, an interspecies factor of 2.5 , and a factor of 10 for interindividual variability a health hazard guide value (RW II) of $0.6 \mathrm{mg}$ / $\mathrm{m}^{3}$ propan-1,2-diol is obtained. A health precaution guide value (RW I) of $0.06 \mathrm{mg} / \mathrm{m}^{3}$ is recommended.

\section{Keywords}

Propan-1,2-diol • propylene glycol • irritation • indoor air • guide value

\subsection{Irritation}

Reizwirkungen von PG im Atemtrakt wurden in wenigen Humanstudien nur nach Kurzzeitexposition sowie in tierexperimentellen subakuten und in einer subchronischen Inhalationsstudie untersucht.

Gesunde Probanden (22 Männer und 5 Frauen) wurden im Rahmen einer Notfallübung im Flugsimulator für 1 Minute PG als Rauchsurrogat ausgesetzt [47]. Die PG-Konzentration wurde mit 11 Punktmessungen (jeweils über $1 \mathrm{~min}$ ) sporadisch geprüft. 18 Probanden waren im Mittel gegenüber $220 \mathrm{mg} \mathrm{PG} / \mathrm{m}^{3}$ exponiert, 9 Probanden gegenüber $520 \mathrm{mg}$ $\mathrm{PG} / \mathrm{m}^{3}$; die Messwerte reichten von 176$851 \mathrm{mg}$ PG/m³ (GM: $309 \mathrm{mg} / \mathrm{m}^{3}$ ). Außer $0,03 \mathrm{mg} / \mathrm{m}^{3}$ Formaldehyd wurden keine anderen flüchtigen organischen Ver- bindungen nachgewiesen. Die Probanden wurden sowohl vor als auch nach der Exposition (innerhalb von $15 \mathrm{~min}$ ) medizinisch untersucht. Zur Untersuchung gehörten akustische Rhinometrie, dynamische Spirometrie und ein vom Arzt abgefragter Fragebogen zu Symptomen. Nach der Exposition mit PG wurde eine verminderte Tränenfilm-Stabilität beobachtet und die Probanden berichteten vermehrt Symptome an Augen und im Rachen. Diese Effekte waren ausgeprägter bei den neun Probanden, die gegenüber höheren PG-Konzentrationen exponiert waren. Alle acht Probanden mit einer Atopie-Vorerkrankung berichteten von Rachenreizungen, von den restlichen 19 Teilnehmern waren es nur $28 \%$. Bei der Lungenfunktion nahm das Verhältnis der Einsekundenkapazität $\left(\mathrm{FEV}_{1}\right)$ zur 
forcierten Vitalkapazität (FVC) leicht ab $(p=0,049)$. Vier der 27 Probanden entwickelten einen Reizhusten und ihre $\mathrm{FEV}_{1}$ sank um 5\%. Aus den publizierten Arbeitsplatzstudien [27, 40, 42], in denen Theater- bzw. Eventnebel als Ursache für Hals-, Nasen- und Atemtrakt-Symptome oder Stimmbandentzündungen untersucht wurden, kann keine Assoziation zu PG hergestellt werden, da diese Nebel stets eine Mischung verschiedener Glykole oder Mineralöle enthielten.

In einer subakuten Inhalationsstudie über 4 Wochen an Sprague-Dawley-Ratten ( $n=15 /$ Geschlecht/Dosis) diente PG als Lösemittel für die Testsubstanz und entsprechend wurden zwei Kontrollgruppen (mit bzw. ohne PG) mitgeführt. Die Tiere waren täglich 1,5 Stunden (bzw. 3 Stunden an den ersten 2-3 Tagen) gegenüber $15.300 \mathrm{mg}$ PG/m $\mathrm{m}^{3}$ (MMAD: 2,7-3,2 $\mu \mathrm{m}$ ) exponiert. Bei den hämatologischen und klinisch-chemischen Untersuchungen ergaben sich keine Unterschiede zwischen den beiden Kontrollgruppen [43].

Werley et al. (2011) [44] führten subakute Studien an Sprague-Dawley-Ratten und Beagles gegenüber PG-Aerosolen durch. Nach einer Kurzzeitexposition von Ratten ( $n=5 /$ Geschlecht/Dosis) für 4 Stunden pro Tag über 7 Tage gegenüber 0 , 21.000 oder $41.000 \mathrm{mg}$ PG $/ \mathrm{m}^{3}$ zeigten sich bei den Tieren keine Effekte. In einer subakuten Folgestudie über 4 Wochen wurden Ratten ( $n=10 /$ Geschlecht/Dosis) für 0,4 , 12, 40 bzw. 120 Minuten am Tag $30.000 \mathrm{mg}$ $\mathrm{PG} / \mathrm{m}^{3}$ ausgesetzt. Bei beiden Geschlechtern war in der Expositionsgruppe ab 40 $\mathrm{min} /$ Tag die Zahl der squamösen Metaplasien im Kehlkopf erhöht und ab 2 Stunden/ Tag signifikant erhöht. In der subakuten Hunde-Studie ( $n=4 /$ Geschlecht/Dosis) wurden die Tiere $0,6,12,36$ oder zweimal 37-49 Minuten am Tag einer Konzentration von $5000 \mathrm{mg}$ PG/m³ exponiert. Bei den weiblichen Tieren stellte man signifikant erniedrigte Hämoglobin- und Hämatokrit-Werte ab einer Exposition von 12 min und eine verringerte Zahl der roten Blutkörperchen ab 36 min fest. Bei den Männchen waren die ebenfalls beobachteten Effekte jedoch nicht signifikant.

Suber et al. (1989) [39] führten eine subchronische Inhalationsstudie an SpragueDawley-Ratten ( $n=19 /$ Geschlecht/Dosis) durch $\left(0,160,1010\right.$ und $2180 \mathrm{mg} P G / \mathrm{m}^{3}$,
6 Stunden/Tag, 5 Tage/Woche über 13 Wochen, „nose-only“). Nach 2 Wochen zeigten sich in der niedrigsten Dosisgruppe bereits bei $70 \%$ der männlichen und bei $65 \%$ der weiblichen Tiere nasale Blutungen. Ab 1010 mg PG/m³ hatten $97-100 \%$ der Tiere am Studienende Nasenblutungen. Hinsichtlich des Wirkungsmechanismus wird vermutet, dass das bei der Metabolisierung von PG in der Nase entstehende Aldehyd 2-Hydroxypropanal, das dann weiter zur 2-Hydroxypropansäure umgesetzt wird, möglicherweise für die nasalen Blutungen verantwortlich ist [10]. Mit steigender PG-Konzentration reagierten auch immer mehr Tiere mit Tränenfluss. Dieser Effekt war dosisabhängig und bei den männlichen Ratten etwas stärker ausgeprägt (m: 5\%/16\%/40\%/40\%; w: $8 \% / 14 \% / 28 \% / 35 \%)$. Weitere dosisabhängige Effekte wurden in den hämatologischen Untersuchungen beobachtet: bei den Weibchen nahmen die Leukozyten, Lymphozyten und stabkernigen Neutrophilen ab (ab der mittleren Dosis signifikant) sowie bei den Männchen die stabkernigen Neutrophilen (in der höchsten Dosis signifikant). In den Serum-Analysen fand man bei den Männchen eine dosisabhängige Erniedrigung der Gammaglutamyltransferase (in der höchsten Dosis signifikant) und von Albumin (ab der mittleren Dosis signifikant), Gesamtprotein und Cholesterin (in der höchsten Dosis signifikant). Bei den histopathologischen Untersuchungen wurden bei den Tieren der mittleren und höchsten Dosis Verdickungen des respiratorischen Epitheliums der Nasenhöhlen festgestellt. Diese Verdickungen können eine Folge der Zunahme der Becherzellen oder des Mucingehaltes in den Becherzellen sein. In der Hochdosisgruppe der Weibchen wurde ein leicht, aber signifikant reduziertes Körpergewicht beobachtet, das mit der verminderten Futteraufnahme ab dem 43. Tag korrelierte, sowie ein signifikant verringertes relatives Lungengewicht. In der Hochdosisgruppe der Männchen fand man ein signifikant erniedrigtes absolutes und relatives Milzgewicht in der höchsten Dosis. Geschädigte Becherzellen wurden bei Kaninchen in der Trachea beobachtet. In der Studie wurden jeweils drei Kaninchen für 20 bzw. 120 min mit PG-Aerosol (10\%ige PG -Lösung) exponiert und der Anteil der geschädigten
Becherzellen stieg mit der Expositionszeit von $53 \%$ auf $69 \%$. Angaben zur Luftkonzentration und eine Kontrollgruppe fehlen [24].

Robertson et al. (1947) [33] exponierten Ratten über 18 Monate mit übersättigtem und Rhesusaffen (12 Monate) mit übersättigtem oder mit ca. $60 \%$ gesättigtem $\left(100-220 \mathrm{mg} / \mathrm{m}^{3}\right)$ PG-Dampf. Die Studie kann aufgrund methodischer Mängel, nicht genauer Bestimmung der Expositionskonzentrationen und relevanter Vorerkrankungen in der Affengruppe nicht für eine Bewertung herangezogen werden.

Relevante chronische Studien mit PG liegen nur für die orale Applikationsroute an Ratten vor. Außer einer leichten Reduktion des Körpergewichtes wurden keine adversen Effekte bis zu Dosierungen von ca. $1700 \mathrm{mg}$ PG/kg KG beobachtet [10].

\subsection{Reproduktions- und Entwicklungstoxizität}

Es liegen keine Hinweise auf eine Reproduktions- oder Entwicklungstoxizität von PG vor.

In einer NTP-Studie mit Mäusen (Trinkwasser bis zu 5\% PG) wurden bis zu der Dosis von 10.100 mg PG/kg KG keine Effekte auf die Fruchtbarkeit der Männchen oder Weibchen der ersten oder zweiten Generation festgestellt [41]. Die Entwicklungstoxizität wurde an verschiedenen Spezies in Studien mit Schlundsondengabe untersucht. Eine von ihnen wurde von US-NTP als ausreichend eingestuft, um die Entwicklungstoxizität zu beurteilen. In dieser Studie an Mäusen wurde auch bei der höchsten Dosierung von $10.000 \mathrm{mg}$ PG/kg KG und Tag (von der Schwangerschaft, des Säugens und bis zur 34 Woche) weder Entwicklungs- noch Maternaltoxizität festgestellt [41].

\subsection{Mutagenität und Kanzerogenität}

PG war in allen in vitro- und in vivo-Studien zur Gentoxizität konsistent negativ [41]. Aus den zweijährigen Fütterungsstudien ergaben sich bei Dosierungen bis zu 1700 mg PG/kg KG pro Tag (männliche Ratten), 2100 mg PG/kg KG pro Tag (weibliche Ratten) bzw. 2500 mg PG/kg KG pro Tag (Ratte) keine Hinweise auf ein muta- 
Tab. Derivation of indoor air guide values: key data

\begin{tabular}{|c|c|c|}
\hline \multirow{2}{*}{$\begin{array}{l}\text { Substance } \\
\text { Parameter }\end{array}$} & \multicolumn{2}{|c|}{ Propan-1,2-diol (propylene g } \\
\hline & Value/Descriptor & Dimensi \\
\hline \multicolumn{3}{|l|}{ General Information } \\
\hline \multicolumn{3}{|l|}{ CLP INDEX No } \\
\hline EC No & $200-338-0$ & \\
\hline CAS No & $57-55-6$ & \\
\hline CLP CMR Classification & Not classified & \\
\hline Indoor air guide value status & Final & \\
\hline $\begin{array}{l}\text { Guide value II } \\
\text { (RW II - Health hazard value) }\end{array}$ & 0.60 & \\
\hline $\begin{array}{l}\text { Guide value I } \\
\text { (RW I - Precautionary value) }\end{array}$ & 0.06 & $\mathrm{mg} / \mathrm{m}^{3}$ \\
\hline Conversion factor: $1 \mathrm{ml} / \mathrm{m}^{3}=$ & 3.16 & $\mathrm{mg} / \mathrm{m}^{3}$ \\
\hline Year & 2017 & \\
\hline \multicolumn{3}{|l|}{ Database } \\
\hline Key study/Author(s) (Year) & Suber et al. 1989 & \\
\hline Species & $\begin{array}{l}\text { Sprague-Dawley } \\
\text { rats }\end{array}$ & \\
\hline Route/type of study & Inhalation & \\
\hline Study length & $\begin{array}{l}\text { Subchronic (90 } \\
\text { days) }\end{array}$ & \\
\hline Inhalative exposure duration & $6 \mathrm{~h} / \mathrm{d}, 5 \mathrm{~d} / \mathrm{wk}$ & \\
\hline Critical endpoint & Nasal haemorrhage & \\
\hline Point of departure (POD) & LOAEC & \\
\hline POD Value & 160 & $\mathrm{mg} / \mathrm{m}^{3}$ \\
\hline
\end{tabular}

Assessment factors

Dose-response assessment factor

Adjusted exposure duration factor (time scaling)

Adjusted study length factor

Route-to-route extrapolation factor

Adjusted absorption factor (inhalation/oral)

Interspecies factor

Intraspecies factor

Sensitive population factor

Other adjustment factors

Quality of whole database

Result

Total assessment factor (TAF)

POD/TAF n. a.

5.6

2

n. a.

n. a.

n. a. kinetic

2.5

10

n.a.

n. a. 280

0.60

$\mathrm{mg} / \mathrm{m}^{3}$

$\mathrm{mg} / \mathrm{m}^{3}$ dynamic

general population, kinetic + dynamic

Calculated guide value; guide value II: $0.60 \mathrm{mg} / \mathrm{m}^{3}$

guide value l: $0.06 \mathrm{mg} / \mathrm{m}^{3}$ genes oder krebserzeugendes Potential von PG [41].

\section{Geruchswahrnehmung}

PG wird als praktisch geruchslos beschrieben [3]. Daten zur Geruchswahrnehmungsschwelle sind nicht verfügbar.

\section{Bewertung}

PG ist in der Luft geruchlich unauffällig und weist beim Menschen ein schwaches haut- und augenreizendes Potential auf. In der einzigen tierexperimentellen subchronischen Inhalationsstudie an Ratten führte PG zu Veränderungen des nasalen Epithels und zu nasalen Blutungen. Der genaue Mechanismus hierfür ist nicht bekannt. Es wird postuliert, dass die Bildung von 2-Hydroxypropanal aus PG für die Blutungen ursächlich ist [10].

\subsection{Bestehende Regelungen und Bewertungen}

Vom niederländischen Expertenausschuss für Arbeitsplatzsicherheit (DECOS) wurde ein Arbeitsplatzgrenzwert von $50 \mathrm{mg}$ $\mathrm{PG} / \mathrm{m}^{3}$ als 8-Stunden-Mittelwert für die Summe der gas- und aerosolförmig vorliegenden PG abgeleitet [9]. Nach Ansicht von DECOS zeigte sich in der subchronischen Inhalationsstudie an Ratten von Suber et al. 1989 [39] erst histopathologisch ab $1010 \mathrm{mg}$ PG/ $\mathrm{m}^{3}$, nicht aber bei $160 \mathrm{mg}$ $\mathrm{PG} / \mathrm{m}^{3}$ ein Anstieg der Anzahl an Becherzellen im Bereich der Nasenmuscheln. Der untere Wert wurde von DECOS als NOAEC gewertet. Die in allen Dosisstufen aufgetretenen nasalen Blutungen wurden von DECOS nicht als kritischer Endpunkt gewählt, da dieser Effekt in anderen Studien nicht beobachtet wurde. Auf der Basis der NOAEC wurde unter Verwendung eines Intraspeziesfaktors von 3 der Arbeitsplatzgrenzwert berechnet. Auf einen Interspeziesfaktor wurde verzichtet, da es sich um lokale Effekte im oberen Atemtrakt handelt.

Die Arbeitsstoff-Kommission der Deutschen Forschungsgemeinschaft sieht hingegen $160 \mathrm{mg}$ PG $/ \mathrm{m}^{3}$ als LOAEC an. Nach ihrer Auffassung könnte ein möglicher Arbeitsplatzgrenzwert für PG im Bereich von $6-12 \mathrm{mg} / \mathrm{m}^{3}$ liegen [10]. Wegen des Fehlens einer NOAEC in der Studie 
von Suber et al. 1989 [39] wurde jedoch kein MAK-Werte abgeleitet.

Die US-amerikanische Agency for Toxic Substances and Disease Registry hat PG 1997 und 2008 bewertet und ein minimal risk level (MRL) von 0,03 mg PG/ $\mathrm{m}^{3}$ für eine intermediäre inhalative Exposition (15-364 Tage) abgeleitet [3, 4]. Der Wert basiert ebenfalls auf den Ergebnissen der Studie von Suber et al. 1989 [39]. Die nasalen Blutungen ab 160 mg PG/m wurden dabei als LOAEC gewertet. Der MRL ergab sich unter Berücksichtigung eines Extrapolationsfaktors von insgesamt 1000 (10 für LOAEC statt NOAEC, 10 als Intraspeziesfaktor und 10 als Interspeziesfaktor) und einer Anpassung an eine kontinuierliche Exposition.

Das JECFA hat eine tägliche duldbare Aufnahmemenge (ADI) von 25 mg PG/kg KG, die zuerst 1973 abgeleitet worden war, im Jahr 2001 bestätigt [23].

Das niederländische Reichsinstitut für Volksgesundheit und Milieuhygiene (RIVM) hat 2016 das Gesundheitsrisiko durch E-Zigaretten für Passivdampfer bewertet. Zur Abschätzung des Gesundheitsrisikos von PG wurde als Schlüsselstudie ebenfalls die Studie von Suber et al. 1989 [39] gewählt und die nasalen Blutungen mit dem LOAEC von $160 \mathrm{mg} / \mathrm{m}^{3}$ wurden als relevanter Endpunkt für die Risikobewertung herangezogen [32].

\subsection{Ableitung von Richtwerten für PG in der Innenraumluft}

Gemäß Basisschema [1] sind zur Ableitung von Richtwerten für die Innenraumluft vorrangig Humanstudien heranzuziehen. Die beiden einzigen bislang publizierten Studien $[46,47]$ können jedoch hierfür nicht berücksichtigt werden. In der Untersuchung von Wieslander \& Norbäck (2010) [46] wurden Arbeiter untersucht, die gleichzeitig verschiedenen anderen Stoffen ausgesetzt waren. In der anderen Untersuchung wurden zwei Gruppen gesunder Probanden einmalig für 1 Minute exponiert und die PG-Konzentrationen nur sporadisch geprüft [47].

Vor diesem Hintergrund wird die tierexperimentelle Studie von Suber et al. (1989) [39] zur Richtwertableitung herangezogen. Hierbei handelt es sich um eine subchronische Inhalationsstudie, in der
Sprague-Dawley-Ratten 6 Stunden pro Tag, 5 Tage pro Woche über 90 Tage PGAerosolen von 0, 160, 1010 oder 2180 mg/ $\mathrm{m}^{3}$ ausgesetzt waren. Für die Festsetzung des Richtwertes II ist nach dem Basisschema von einer LOAEC, also der niedrigsten beobachteten adversen Wirkkonzentration, auszugehen [1].

Für PG wird auf der Basis der vorgenannten Studie die bei $160 \mathrm{mg} / \mathrm{m}^{3}$ beobachteten nasalen Blutungen als für die $\mathrm{Ab}$ leitung des Richtwerts II kritischer Effekt gesehen.

Gemäß Basisschema werden folgende Standard-Extrapolationsfaktoren berücksichtigt:

- Faktor 5,6 zur Extrapolation auf eine kontinuierliche Exposition (von $6 \mathrm{~h} / \mathrm{d}$ und $5 \mathrm{~d}$ /Woche auf $24 \mathrm{~h} /$ Tag und 7 Tage/Woche).

- Faktor 2 zur Extrapolation von subchronischer auf chronische Exposition.

- Es wird vermutet, dass die Bildung des Aldehyds 2-Hydroxypropanal für die nasalen Blutungen verantwortlich sein könnte. Die lokale Aldehyd-Konzentration hängt sowohl von der nasalen ADH- als auch ALDH-Aktivität ab. Verschiedene Studien konnten zeigen, dass Speziesunterschiede der ALDH-Aktivität im Atemtrakt vorliegen [7, 21, 22]. Für den nasalen Metabolismus von PG selbst liegen jedoch keine Daten vor. Daher zieht der AIR zur Berücksichtigung von Interspeziesunterschieden bzgl. des lokalen Metabolismus den Faktor von 2,5 heran.

- Faktor 10 zur Berücksichtigung der interindividuellen Variabilität [1],

- Angesichts der tierexperimentell beobachteten Effekte ist der obere Atemtrakt das empfindlichste Zielorgan, so dass die lokale Konzentration von PG bzw. dessen Metaboliten den Effekt bestimmt. Da anthropometrische Expositionsmodelle keine wesentlichen Unterschiede in der lokalen Deposition von gasförmigen Substanzen im oberen Atemtrakt zwischen Kindern und Erwachsenen zeigten [16, 17] und die vorhandenen Daten zur Enzymausstattung und -aktivität von Kindern keinen nachteiligen Effekt erwarten lassen [13, 48], hält der AIR einen zusätzlichen Faktor zur Berück- sichtigung der besonderen Empfindlichkeit von Kindern gegenüber PG für nicht notwendig.

Somit ergibt sich $160 \mathrm{mg} / \mathrm{m}^{3}: 280=0,6 \mathrm{mg} /$ $\mathrm{m}^{3}$.

Der AIR legt als Richtwert II eine PGKonzentration von $0,6 \mathrm{mg} / \mathrm{m}^{3}$ fest.

Zur Ableitung eines RW I hält der AIR angesichts des Fehlens einer NOAEC in der subchronischen Inhalationsstudie einen Faktor von 10 für angemessen. Er legt daher einen Richtwert I von 0,06 mg PG/ $\mathrm{m}^{3}$ fest.

\section{Anmerkungen}

Der Textentwurf dieser Mitteilung wurde von Prof. Dr. Hermann Fromme, Dr. Katrin Schröder und Dr. Helmut Sagunski erstellt und vom Ausschuss für Innenraumrichtwerte (AIR) im März 2017 verabschiedet. Die Literaturrecherche wurde im März 2017 abgeschlossen.

\section{Literatur}

1. Ad-hoc-AG (2012) Richtwerte für die Innenraumluft: erste Fortschreibung des Basisschemas. Mitteilung der Ad-hoc-Arbeitsgruppe Innenraumrichtwerte der IRK und AOLG. Bundesgesundheitsbl 55:279-290

2. Arbour R, Esparis B (2000) Osmolar gap metabolic acidosis in a 60-year-old man treated for hypoxemic respiratory failure. Chest 118:545-546

3. ATSDR (Agency for Toxic Substances and Disease Registry, USA) (1997) Toxicological profile for propylene glycol. http://www.atsdr.cdc.gov/ ToxProfiles/tp189.pdf. Zugegriffen: 1. März 2017

4. ATSDR (2008) Addendum to the toxicological profile for propylene glycol. Atlanta https://www. atsdr.cdc.gov/toxprofiles/propylene_glycol_addendum.pdf. Zugegriffen: 1. März 2017

5. Bau SK, Aspin N, Wood D, Levison H (1971) The measurement of fluid deposition in humans following mist tent therapy. Pediatrics 48:605-612

6. BAUA (Federal Institute for Occupational Safety and Health) (2015) CLH report. Proposal for harmonised classification and labelling. Substance name: Propane-1,2-diol. Dortmund

7. Bogdanffy MS, Sarangapani R, Kimbell JS, Frame SR, Plowchalk DR (1998) Analysis of vinyl acetate metabolism in rat and human nasal tissues by an in vitro gas uptake technique. Toxicol Sci 46(2):235-246

8. Choi H, Schmidbauer N, Spengler J, Bornehag CG (2010) Sources of propylene glycol and glycol ethers in air at home. Int J Environ Res Public Health 7:4213-4237

9. DECOS (Dutch Expert Committee on Occupational) (2007) Propylene glycol (1,2-propanediol); Healthbased recommended occupational exposure limit. 
The Hague: Health Council of the Netherlands. Publication no. 2007/020SH.

10. DFG (2007) Propylenglykol MAK Value Documentation in German language, 2007.http:// onlinelibrary.wiley.com/doi/10.1002/3527600418. mb5755kskd0042/pdf. Zugegriffen: 1. März 2017

11. EC (European Commission) (2011) EC Regulation No 1130/2011. Amending Annex III to Regulation (EC) No 1333/2008 of the European Parliament and of the Council on food additives by establishing a Union list of food additives approved for use in food additives, food enzymes, food flavourings and nutrients. Off J Eur Union L 295:178 (Online: http://eur-lex.europa.eu/LexUriServ/ LexUriServ.do?uri=OJ:L:2011:295:0178:0204:EN:P DF)

12. Fiume MM, Bergfeld WF, Belsito DV, Hill RA, Klaassen CD, Liebler D, Marks JG Jr, Shank RC, Slaga TJ, Snyder PW, Andersen FA (2012) Safety assessment of propylene glycol, tripropylene glycol, and PPGs as used in cosmetics. Int J Toxicol 31(5 Suppl):245S-260S

13. Schneider K, Gerdes H, Hassauer M, Oltmanns J, Schulze J (2002) Berücksichtigung der Risikogruppe Kind bei der Ableitung gesundheitsbezogener Umweltstandards. Bericht des Forschungs- und Beratungsinstitutes Gefahrstoffe $\mathrm{GmbH}$ (FoBiG).

14. Fromme $H$, Heitmann D, Dietrich $S$, Schierl $R$, Körner W, Kiranoglu M, Zapf A, Twardella D (2008) Raumluftqualität in Schulen - Belastung von Klassenräumen mit Kohlendioxid (CO2), flüchtigen organischen Verbindungen (VOC), Aldehyden, Endotoxinen und Katzenallergenen. Gesundheitswesen 70:88-97

15. Geiss O, Bianchi I, Barahona F, Barrero-Moreno J (2015) Characterisation of mainstream and passive vapours emitted by selected electronic cigarettes. Int J Hyg Environ Health 218:169-180

16. Ginsberg GL, Perkovich Foos B, Firestone MP (2005) Review and analysis of inhalation dosimetry methods for application to children's risk assessment. J Toxicol Environ Health Part A 68:573-615

17. Ginsberg G, Foos B, Dzubow RB, Firestone M (2010) Options for incorporating children's inhaled dose into human health risk assessment. Inhal Toxicol 22:627-647

18. Hodgson AT, Rudd AF, Beal D, Chandra S (2000) Volatile organic compound concentrations and emission rates in new manufactured and site-built houses. Indoor Air 10:178-192

19. Hofmann H, Erdmann G, Müller A (2014) Zielkonflikt energieeffiziente Bauweise und gute Raumluftqualität - Datenerhebung für flüchtige organische Verbindungen in der Innenraumluft von Wohn- und Bürogebäuden (Lösungswege). Hg.: Arbeitsgemeinschaft Ökologischer Forschungsinstitute e. V. Anhang. http://www. agoef.de/fileadmin/user_upload/dokumente/ forschung/AGOEF-Abschlussericht_VOCDB_II_Anhang-nicht-barrierefrei.pdf

20. Ishiwata H, Yamada T, Yoshiike N, Nishijima M, Kawamoto A, Uyama Y (2002) Daily intake of food additives in Japan in five age groups estimated by the market basket method. Eur Food Res Technol 215:367-374

21. Ito $Y$, Yokota H, Wang R, Yamanoshita O, Ichihara G, Wang H, Kurata Y, Takagi K, Nakajima T (2005) Species differences in the metabolism of di(2-ethylhexyl) phthalate (DEHP) in several organs of mice, rats, and marmosets. Arch Toxicol 79(3):147-154

22. Ito $Y$, Kamijima M, Hasegawa C, Tagawa M, Kawai T, Miyake M, Hayashi Y, Naito H, Nakajima T (2014) Species and inter-individual differences in meta- bolic capacity of di(2-ethylhexyl)phthalate (DEHP) between human and mouse livers. Environ Health Prev Med 19(2):117-125

23. JECFA (Joint FAO/WHO Expert Committee on Food Additives) (2002) Evaluation of certain food additives and contaminants: fifty-seventh report of the Joint FAO/WHO expert committee on food additives.

24. Konrádová V, Vavrova V, Janota J (1978) Effect of the inhalation of a surface tension-reducing substance (propylene glycol) on the ultrastructure of epithelium of the respiratory passages in rabbits. Folia Morphol (Praha) 26:28-34

25. Lim HS, Hwang JY, Choi E, Lee GY, Yun SS, Kang T (2016) Assessment of the dietary intake of propylene glycol in the Korean population. Food Addit Contam Part A 33:1290-1298

26. McAuley TR, Hopke PK, Zhao J, Babaian S (2012) Comparison of the effects of e-cigarette vapor and cigarette smoke on indoor air quality. Inhal Toxicol 24:850-857

27. Moline JM, Golden AL, Highland JH, Wilmarth KR, Kao AS (2000) Health effects evaluation of theatrical smoke, haze and pyrotechnics. Prepared for Equity-Leage Pension and Health Trust Funds, 6. Juni 2000.

28. Morshed KM, Nagpaul JP, Majumdar S, Amma MK (1988) Kinetics of propylene glycol elimination and metabolism in rat. Biochem Med Metab Biol 39:90-97

29. Morshed KM, L'Helgoualch A, Nagpaul JP, Amma MK, Desjeux JF (1991) The role of propylene glycol metabolism in lactatemia in the rabbit. Biochem Med Metab Biol 46:145-151

30. Neumann H-D, Hahn J-U, Assenmacher-Maiworm H, Birtel H, Kussin H (1996) Belastung durch Nebelfluide zur szenischen Gestaltung auf Bühnen. Gefahrst Reinhalt Luft 56:431-436

31. O'Connell G, Colard S, Cahours X, Pritchard JD (2015) An Assessment of Indoor Air Quality before, during and after Unrestricted Use of E-Cigarettes in a Small Room. Int J Environ Res Public Health 12:4889-4907

32. RIVM (2016) De gezondheidsrisico's van esigaretten. RIVM Briefrapport 2016-0036. http:// nl.sitestat.com/rivm/rivm-nl/s?link.documenten en_publicaties.wetenschappelijk.rapporten.2016. juli.de_gezondheidsrisico_s_van_e_sigaretten_voor_omstanders.download_pdf\&ns type=pdf\&ns_url=http\%3A\%2F\%2Fwww.rivm.nl \%2Fdsresource\%3Fobjectid=rivmp:319228\&type $=$ org\&disposition=inline\&ns_nc=1. Zugegriffen: 1 . März 2017

33. Robertson OH, Loosli CG, Puck TT, Wise H, Lemon HM, Lester W Jr (1947) Test for the chronic toxicity of propylene glycol on monkeys and rats by vapor inhalation and oral administration. J Pharmacol Exp Ther 91:52-76

34. Salonen $H$, Pasanen AL, Lappalainen S, Riuttala H, Tuomi T, Pasanen P, Back B, Reijula K (2009) Volatile organic compounds and formaldehyde as explaining factors for sensory irritation in office environments. J Occup Environ Hyg 6:239-247

35. Schleibinger $\mathrm{H}$, Hott U, Marchl D, Braun B, Plieninger $P$, Rüden $H$ (2001) VOC-Konzentrationen in Innenräumen des Großraums Berlin im Zeitraum von 1988-1999. Gefahrst Reinhalt Luft 61:26-38

36. Schober W, Szendrei K, Matzen W, Osiander-Fuchs H, Heitmann D, Schettgen T, Jörres RA, Fromme H (2014) Use of electronic cigarettes (e-cigarettes) impairs indoor air quality and increases FeNO levels of e-cigarette consumers. Int J Hyg Environ Health 217:628-637
37. Schripp T, Markewitz D, Uhde E, Salthammer T (2013) Does e-cigarette consumption cause passive vaping? Indoor Air 23:25-31

38. Speth PA, Vree TB, Neilen NF, de Mulder PH, Newell DR, Gore ME, de Pauw BE (1987) Propylene glycol pharmacokinetics and effects after intravenous infusion in humans. Ther Drug Monit 9:255-258

39. Suber RL, Deskin R, Nikiforov I, Fouillet X, Coggins CR (1989) Subchronic nose-only inhalation study of propylene glycol in Sprague-Dawley rats. Food Chem Toxicol 27:573-583

40. US-NIOSH (National Institute for Occupational Safety and Health) (1994) Health Hazard Evaluation Report. HETA 90-0355-2449.https://www.cdc. gov/niosh/hhe/reports/pdfs/1990-0355-2449.pdf. Zugegriffen: 1. März 2017

41. US-NTP-CERHR (2004) (National Toxicology Program-Center for the Evaluation of Risks to Human Reproduction) Expert Panel report on the reproductive and developmental toxicity of propylene glycol. Reproduct Toxicol 18:533-579

42. Varughese $S$, Teschke $K$, Brauer M, Chow $Y$, van Netten C, Kennedy SM (2005) Effects of theatrical smokes and fogs on respiratory health in the entertainment industry. Am J Ind Med 47(5):411-418

43. Wang $T$, Noonberg $S$, Steigerwalt R, Lynch M, Kovelesky RA, Rodríguez CA, Sprugel K, Turner N (2007) Preclinical safety evaluation of inhaled cyclosporine in propylene glycol. J Aerosol Med 20(4):417-428

44. Werley MS, McDonald P, Lilly P, Kirkpatrick D, Wallery J, Byron P, Venitz J (2011) Non-clinical safety and pharmacokinetic evaluations of propylene glycol aerosol in Sprague-Dawley rats and Beagle dogs. Toxicol 287:76-90

45. West R, Banton M, Hu J, Klapacz J (2014) The distribution, fate, and effects of propylene glycol substances in the environment. Rev Environ Contam Toxicol 232:107-138

46. Wieslander G, Norbäck D (2010) Ocular symptoms, tear film stability, nasal patency, and biomarkers in nasal lavage in indoor painters in relation to emissions from water-based paint. Int Arch Occup Environ Health 83:733-741

47. Wieslander G, Norbäck D, Lindgren T (2001) Experimental exposure to propylene glycol mist in aviation emergency training: acute ocular and respiratory effects. Occup Environ Med 58:649-655

48. Yoon M, Madden MC, Barton HA (2006) Developmental expression of aldehyde dehydrogenase in rat: a comparison of liver and lung development. Toxicol Sci 89:386-398

49. Yu DK, Elmquist WF, Sawchuk RJ (1985) Pharmacokinetics of propylene glycol in humans during multiple dosing regimens. J Pharm Sci 74:876-879

50. Yu DK, Sawchuk RJ (1987) Pharmacokinetics of propylene glycol in the rabbit. J Pharmacokinet Biopharm 15:453-471

51. Zhu J, Feng YL, Aikawa B (2004) A positive chemical ionization GC/MS method for the determination of airborne ethylene glycol and propylene glycols in non-occupational environments. J Environ Monit 6:881-887 